\title{
Effects of hypoxia ischemia on caspase-3 expression and neuronal apoptosis in the brain of neonatal mice
}

\author{
CHANGBO DENG, JUAN LI, LUYI LI, FENGJIE SUN and JIQING XIE \\ Department of Pediatrics, The Fifth Affiliated Hospital of Guangzhou Medical University, \\ Guangzhou, Guangdong 510700, P.R. China
}

Received June 18, 2018; Accepted February 1, 2019

DOI: $10.3892 /$ etm.2019.7487

\begin{abstract}
Effects of hypoxia ischemia on caspase-3 expression and neuronal apoptosis in the brain of neonatal mice were investigated. Twenty-five neonatal CD1 mice aged 1 week were selected and randomly divided into sham-operation group $(n=8)$ and newborn hypoxia ischemia encephalopathy (NHIE) model group $(n=17)$. The messenger ribonucleic acid (mRNA) expression levels of caspase-3 and Fas ligand (FasL) in brain tissues of mice in both groups were detected via reverse transcription-polymerase chain reaction (RT-PCR). The protein expression levels of caspase-3 and FasL in mice in both groups were detected via western blotting. Moreover, apoptosis of brain tissues was detected using the terminal deoxynucleotidyl transferase-mediated dUTP nick end labeling (TUNEL), and caspase-3 protein expression level in brain tissues was detected using immunohistochemical methods. Results of RT-PCR and western blotting revealed that compared with those in sham-operation group, caspase-3 and FasL expression levels in model group were significantly increased. Results of TUNEL showed that the number of apoptotic neurons in model group was significantly increased. Besides, results of immunohistochemical detection manifested that the caspase-3 protein expression level in model group was obviously increased. Hypoxia ischemia can lead to significant increase of caspase-3 expression and increase of neuronal apoptosis in the brain of neonatal mice.
\end{abstract}

\section{Introduction}

Neonatal hypoxic-ischemic brain damage (HIBD) can cause a variety of serious irreversible neurological sequelae, including cerebral palsy, epilepsy, delayed growth and development, cognitive impairment, intelligence disturbance, and even

Correspondence to: Dr Changbo Deng, Department of Pediatrics, The Fifth Affiliated Hospital of Guangzhou Medical University, 621 Gangwan Road, Huangpu, Guangzhou, Guangdong 510700, P.R. China

E-mail: z483kyg@163.com

Key words: hypoxia ischemia, caspase-3, neuronal apoptosis neonatal death (1). According to a large number of studies, the incidence rate of asphyxia in neonates during delivery is as high as 5\% in China. Hypoxia ischemia encephalopathy occurs in approximately 100,000 neonates every year due to severe asphyxia during delivery $(1,2)$. However, there have been no effective means to improve a variety of severe neurological sequelae caused by perinatal hypoxia ischemia. Therefore, research and investigation of neonatal HIBD is of profound significance.

The pathogenesis of HIBD involves a wide range of pathological mechanism, which is the result of a series of lesions caused by various factors (3). It has been found that neuronal apoptosis plays an important role in the pathologic process of HIBD, which is closely related to the pathologic degree of HIBD. A large number of studies have confirmed that neuronal apoptosis is involved and plays an important role in the development process of immature brain and in the process of nervous system injury, such as cerebral hypoxia, ischemia and trauma $(4,5)$. Apoptosis involves a variety of factors, in which the caspase family plays a dominant role. Caspase-3, also known as the executing molecule that exerts an apoptotic function in various apoptotic pathways, is studied the most and clarified the most clearly (6-8). In this study, the mouse model of HIBD was constructed to study neuronal apoptosis and caspase-3 expression in brain tissues in HIBD, so as to lay an experimental foundation for further study on the mechanism of drugs.

\section{Materials and methods}

Materials. A total of 25 neonatal CD1 mice aged 1 week were purchased from Nanjing Jiancheng Laboratory Animal Co., Ltd. (Nanjing, China). Terminal deoxynucleotidyl transferasemediated dUTP nick end labeling (TUNEL) apoptosis assay kit (S7165; Merck KGaA, Darmstadt, Germany), rabbit anti-mouse Fas ligand (FasL), caspase-3 and glyceraldehyde-3-phosphate dehydrogenase (GAPDH) primary antibodies and horseradish peroxidase-labeled secondary antibody (Proteintech, Wuhan, China), reverse transcription-polymerase chain reaction (RT-PCR) kit (Ambion; Thermo Fisher Scientific, Inc., Waltham, MA, USA), DNA polymerase (Sigma-Aldrich, St. Louis, MO, USA), immunohistochemical staining kit SP-9001 (Beijing Zhongshan Goldenbridge Biotechnology Co., Ltd., Beijing, China), FasL and caspase-3 primers (synthesized 
by Shanghai GenePharma Co., Ltd., Shanghai, China), and Leica DMi8 fluorescence microscope (Leica Microsystems $\mathrm{GmbH}$, Wetzlar, Germany) and ImageJ analysis software (NIH, Bethesda, MD, USA).

The present study was approved by the Ethics Committee of The Fifth Affiliated Hospital of Guangzhou Medical University (Guangzhou, China).

Construction of mouse model of newborn hypoxia ischemia encephalopathy (NHIE). Neonatal male CD1 mice (70-90 g) were randomly divided into sham-operation group $(n=8)$ and NHIE model group $(n=17)$. Mice in NHIE model group were treated as follows: First, after anesthesia via inhalation of isoflurane, a cervical median incision was made, the right common carotid artery was correctly separated and closed via electrocoagulation, and then the incision was sutured. After operation, mice were revived at room temperature, normally fed with breast milk and treated under hypoxia: NHIE mice were placed into a closed chamber at $37^{\circ} \mathrm{C}$ injected with nitrogen gas containing $5 \% \mathrm{CO}_{2}$ and $8 \% \mathrm{O}_{2}$. After $100 \mathrm{~min}$, mice were taken and continued to be normally fed with breast milk. In sham-operation group, the incision was sutured after operation without hypoxia treatment and closure of common carotid artery.

Detection of caspase-3 and FasL mRNA expression levels in brain tissues. The total RNA was extracted from brain tissues using TRIzol (Beyotime, Shanghai, China). After that, $1 \mu \mathrm{g}$ RNA was taken from each group and reversely transcribed into complementary DNA (cDNA) according to instructions of the kit. Then caspase- 3 and FasL primers were added, and caspase-3 and FasL mRNA expression levels were quantitatively detected via PCR, with GAPDH as the internal reference. Primer sequences of caspase-3, FasL and GAPDH are shown in Table I. Reaction conditions were as follows: at $94^{\circ} \mathrm{C}$ for $5 \mathrm{~min}$, at $94^{\circ} \mathrm{C}$ for $30 \mathrm{sec}$, at $57^{\circ} \mathrm{C}$ for $30 \mathrm{sec}$, at $72^{\circ} \mathrm{C}$ for $30 \mathrm{sec}$, amplification for 30 cycles, and at $72^{\circ} \mathrm{C}$ for $5 \mathrm{~min}$.

Detection of caspase-3 and FasL protein expression levels in brain tissues via western blotting. Brain tissues were taken to extract the total protein according to instructions of the protein extraction kit, and the protein concentration was determined using bicinchoninic acid (BCA) method, followed by $10 \%$ sodium dodecyl sulfate polyacrylamide gel electrophoresis (SDS-PAGE). After electrophoresis, the protein was transferred onto a polyvinylidene fluoride (PVDF) membrane using the wet method, sealed with skim milk, and incubated with primary rabbit anti-mouse caspase-3, FasL and GAPDH monoclonal antibodies (dilution, 1:1,000; cat. nos. 19677-1-AP, 13098-1-AP and 10494-1-AP; Proteintech) at $4^{\circ} \mathrm{C}$ for $16 \mathrm{~h}$. After the membrane was washed with TTBS, the HRP-labeled secondary goat anti-rabbit polyclonal antibody (dilution, 1:1,200; cat. nos. SA00001-2; Proteintech) was added and the mixture was shaken at $20^{\circ} \mathrm{C}$ for $2 \mathrm{~h}$, followed by image development via electrochemiluminescence (ECL) in a darkroom and photography. Finally, the protein band was analyzed using Image Lab 4.0.1 software (Bio-Rad Laboratories, Inc., Hercules, CA, USA).

Immunohistochemical detection of caspase-3 protein expression in brain tissues. The detection was performed as follows
Table I. RT-PCR primer sequences and gene silencing sequences.

Genes $\quad$ Primer sequence

\begin{tabular}{ll}
\hline Caspase-3 & F: 5'-TGCGTGTGGAGTATTTGGATG-3' \\
& R: 5'-TGGTACAGTCAGAGCCAACCTC-3' \\
FasL & F: 5'-TGGGATGCCTTTGTGGAAC-3' \\
& R: 5'-CATATTTGTTTGGGGCAGGTC-3' \\
GAPDH & F: 5'-ATGGCACCGTCAAGGCTGAG-3' \\
& R: 5'-GCAGTGATGGCATGGACTGT-3' \\
\hline
\end{tabular}

F, forward; R, reverse.

according to instructions of the immunohistochemical staining kit SP-9001: After the paraffin-embedded sections were deparaffinized, the endogenous peroxidase activity was eliminated using hydrogen peroxide, and the citrate buffer was used for antigen retrieval. Then sections were sealed with $10 \%$ goat serum and added with primary antibody (diluted at 1:100) in a refrigerator at $4^{\circ} \mathrm{C}$ overnight. After sections were washed with phosphate-buffered saline (PBS), they were incubated with secondary antibody and washed again with PBS 15 min later, followed by color development via diaminobenzidine (DAB), hematoxylin counterstaining and photography under the microscope.

Detection of apoptosis in brain tissues. At 3 days after modeling, mice were anesthetized with $4 \%$ isoflurane, and the brain was immediately taken from 3 mice in each group. The brain was fixed in $4 \%$ paraformaldehyde solution, washed with PBS and sliced into $30 \mu \mathrm{m}$ sections, and then sections were fixed in $1 \%$ paraformaldehyde solution to be used in subsequent experiments. Brain tissue sections (3-5) were randomly taken from each group and treated according to instructions of the TUNEL assay kit, followed by observation and photography under the fluorescence microscope. Red-labeled cells were TUNEL-positive cells, namely apoptotic cells.

Statistical analysis. Data in this study are presented as mean \pm standard deviation (mean \pm SD). SPSS 17.0 software (SPSS Inc., Chicago, IL, USA) and one-way analysis of variance (ANOVA) were adopted for data processing. $\mathrm{P} \leq 0.05$ was considered to indicate a statistically significant difference.

\section{Results}

Caspase-3 and FasL mRNA expression levels in brain tissues. Results of RT-PCR revealed that compared with those in sham-operation group, the caspase-3 and FasL mRNA expression levels in NHIE model group were significantly increased $(\mathrm{P}<0.01)$, indicating that caspase-3 and FasL mRNA expression levels have a certain association with hypoxia ischemia in brain tissues (Fig. 1).

Caspase-3 and FasL protein expression levels in brain tissues. Results of western blotting showed that caspase-3 and FasL protein expression levels in NHIE model group were 

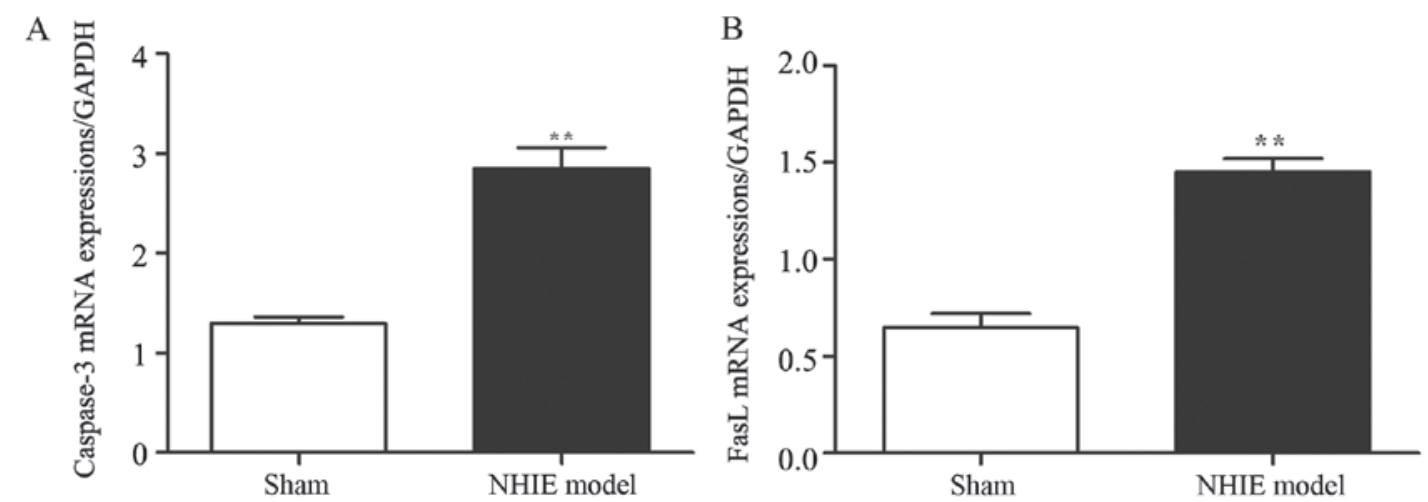

Figure 1. Detection of (A) caspase-3 and (B) FasL mRNA expression in brain tissues via RT-PCR. ${ }^{* *} \mathrm{P}<0.01$ vs. sham-operation group.
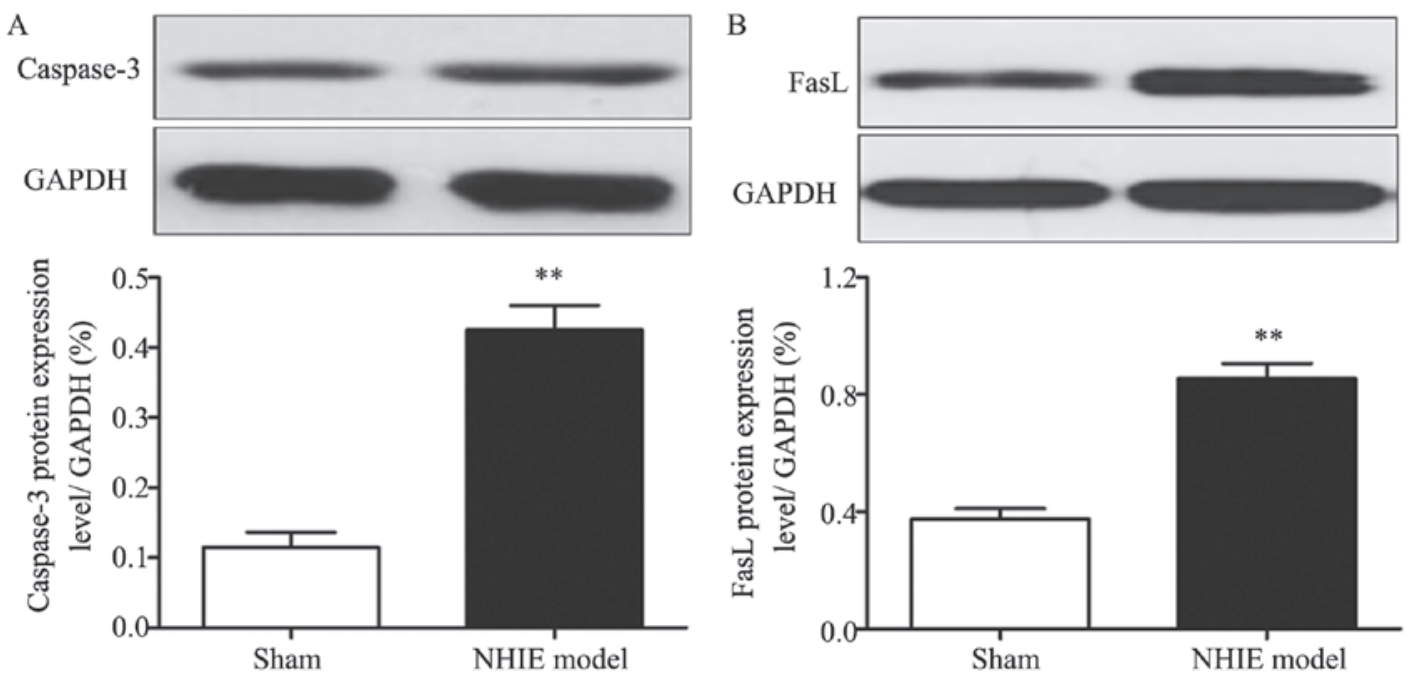

Figure 2. Detection of (A) caspase-3 and (B) FasL protein expression in brain tissues via western blotting. ${ }^{* *} \mathrm{P}<0.01$ vs. sham-operation group.

obviously increased compared with those in sham-operation group $(\mathrm{P}<0.01)$, suggesting that caspase-3 and FasL protein expression levels have a certain association with hypoxia ischemia in brain tissues (Fig. 2).

Detection of neuronal apoptosis in brain tissues via TUNEL. The number of TUNEL-positive cells in brain tissues in NHIE model group was obviously increased compared with that in sham-operation group, indicating that hypoxia ischemia can lead to neuronal apoptosis (Fig. 3).

Immunohistochemical detection of caspase-3 protein expression in brain tissues. Compared with that in shamoperation group, brain tissues in NHIE model group were stained significantly and the caspase-3 protein expression was remarkably increased, suggesting that hypoxia ischemia can lead to the significant upregulation of caspase-3 protein expression (Fig. 4).

\section{Discussion}

At present, neonatal HIBD is still the main cause of neonatal death and a variety of severe neurological sequelae, whose pathogenesis involves various factors and pathological changes. Therefore, there is still a lack of effective prevention and treatment means in clinic.

In the pathological mechanism of neonatal HIBD, important factors leading to neuronal damage include accumulation of excitatory amino acid, oxidative stress and apoptosis. The most serious damage in the process of HIBD is neuronal apoptosis, and caspase- 3 and $\mathrm{Bcl}-2$ are important proteins executing apoptosis, which have significantly increased expression levels in the process of hypoxia ischemia and play important roles in pathogenesis $(9,10)$. The final result of various pathways leading to cell damage is irreversible apoptosis, so protecting neurons and inhibiting apoptosis are the most effective treatment means for neonatal HIBD (11). Reports have demonstrated that the apoptosis process includes transmission of apoptotic signals and execution of apoptosis command. During the signal transmission, the activation of caspase family is realized through the following pathways. In the endogenous pathway, also known as the mitochondrial pathway, cytochrome $c$ in the mitochondria is released into the cytoplasm and interacts with activator-1 to activate caspase-9. In the exogenous pathway, also known as the death receptor pathway, the signaling molecules will activate the death receptor on the surface of cytoplasmic membrane to activate caspase-8. In the endoplasmic reticulum pathway, 

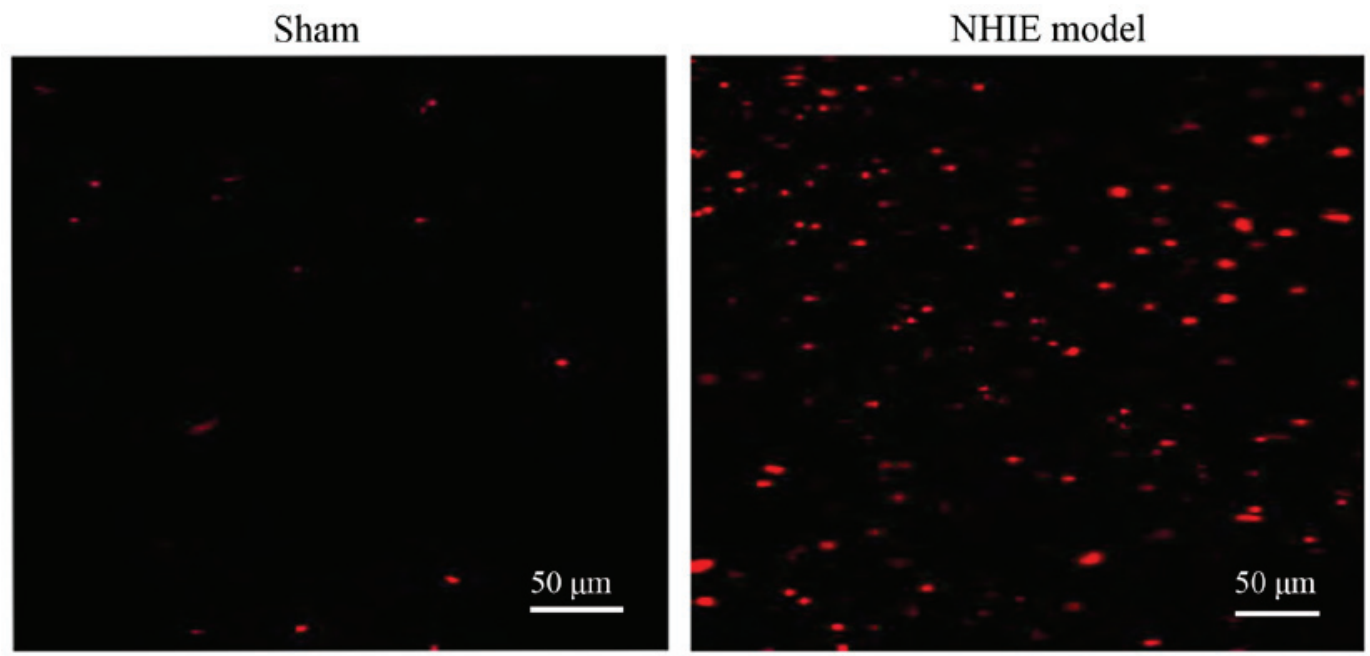

Figure 3. Detection of neuronal apoptosis in brain tissues via TUNEL (x100).
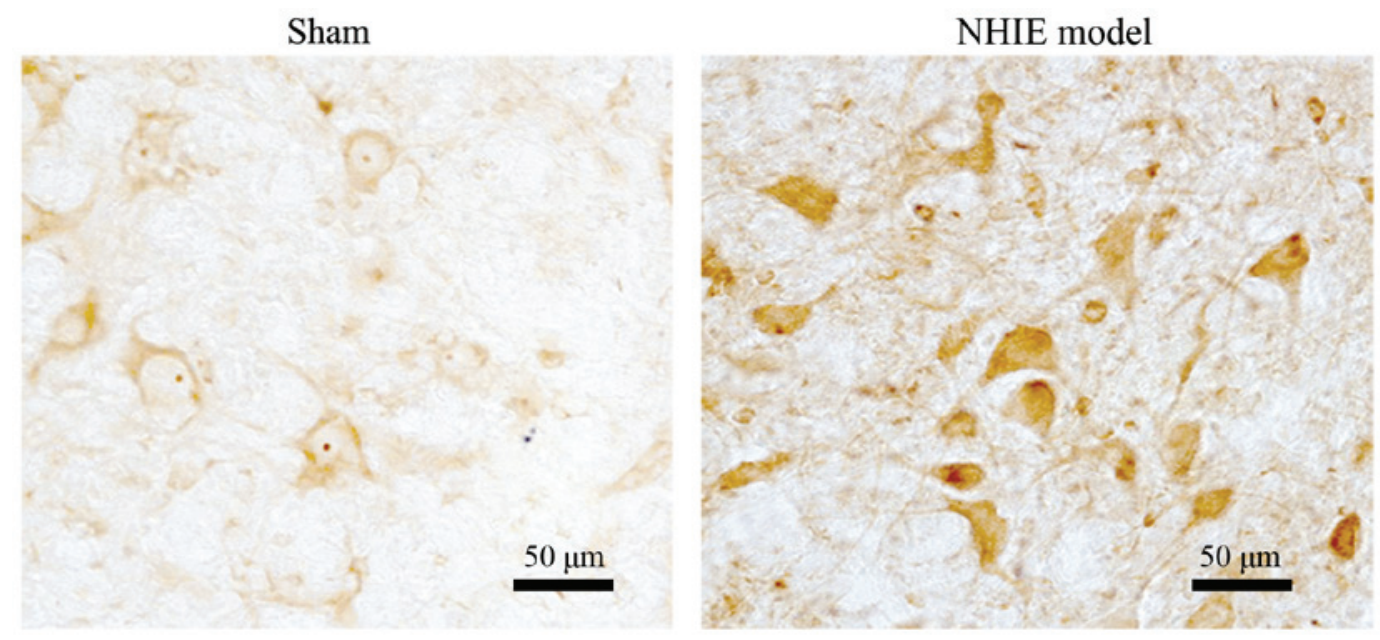

Figure 4. Immunohistochemical detection of caspase-3 protein expression in brain tissues (x400).

endoplasmic reticulum stress will be produced when cells are stimulated, ultimately activating caspase-9 $(12,13)$. The caspase family is the core in the apoptosis process. Caspase is stored in the cytoplasm in the form of zymogen after being synthesized, and a chain reaction occurs after caspase receives related signal commands, so that caspase is cleaved and activated, further leading to signaling cascade amplification and activating a variety of downstream proteases, thereby initiating multiple apoptotic pathways. Cleavage of caspase is a reversible process, while activation of caspase- 3 is an irreversible process, and caspase- 3 is the executor of the final apoptotic signal (14). Therefore, inhibiting activation or reversible stage of caspase-3 can effectively inhibit apoptosis, thereby alleviating the pathological changes of HIBD. In the Fas signaling pathway, Fas and its ligand FasL exert the function, the latter of which, as a transmembrane protein, mediates apoptosis when its expression is increased through binding to Fas (15).

In this study, the mRNA and protein expression levels of caspase-3 and FasL in neonatal mice in model group were significantly increased. TUNEL results manifested that the number of apoptotic neurons in model group was significantly increased. Results of immunohistochemical detection showed that the caspase-3 expression in model group was obviously increased. Similarly, there are reports that the activation and expression of caspase-3 are remarkably increased within a short period of time after intracerebral and extracerebral injury. The activity and protein expression of caspase-3 were downregulated obviously in the experiment about the effect of endogenous caspase inhibitor or exogenous caspase inhibitor on the transcription level $(16,17)$. In addition, some studies have demonstrated that the activity and expression of caspase-3 in brain tissues are significantly increased in neonatal HIBD mice, and inhibiting the caspase-3 expression level can prevent neuronal apoptosis. Therefore, inhibiting caspase-3 expression plays a role in protecting nerve cells (18-20).

In conclusion, the mouse model of neonatal HIBD was constructed in this study to investigate apoptosis and caspase-3 expression in brain tissues, and it was found that hypoxia ischemia could lead to significant increase of caspase-3 expression and increase of neuronal apoptosis in the brain of neonatal mice, thus laying an experimental foundation for further study on the mechanism of drugs. 


\section{Acknowledgements}

Not applicable.

\section{Funding}

No funding was received.

\section{Availability of data and materials}

The datasets used and/or analyzed during the current study are available from the corresponding author on reasonable request.

\section{Authors' contributions}

CD wrote the manuscript. CD and JL performed PCR. LL and FS assisted with animal model construction. JX was responsible for immunohistochemical detection. All authors read and approved the final manuscript.

\section{Ethics approval and consent to participate}

The study was approved by the Ethics Committee of The Fifth Affiliated Hospital of Guangzhou Medical University (Guangzhou, China).

\section{Patient consent for publication}

Not applicable.

\section{Competing interests}

The authors declare that they have no competing interests.

\section{References}

1. Gluckman PD, Wyatt JS, Azzopardi D, Ballard R, Edwards AD, Ferriero DM, Polin RA, Robertson CM, Thoresen M, Whitelaw A, et al: Selective head cooling with mild systemic hypothermia after neonatal encephalopathy: Multicentre randomised trial. Lancet 365: 663-670, 2005.

2. Zheng H, Dai T, Zhou B, Zhu J, Huang H, Wang M and Fu G: SDF-1alpha/CXCR4 decreases endothelial progenitor cells apoptosis under serum deprivation by PI3K/Akt/eNOS pathway. Atherosclerosis 201: 36-42, 2008.

3. Miller JT, Bartley JH, Wimborne HJ, Walker AL, Hess DC, Hill WD and Carroll JE: The neuroblast and angioblast chemotaxic factor SDF-1 (CXCL12) expression is briefly up regulated by reactive astrocytes in brain following neonatal hypoxic-ischemic injury. BMC Neurosci 6: 63, 2005.

4. Northington FJ, Ferriero DM, Flock DL and Martin LJ: Delayed neurodegeneration in neonatal rat thalamus after hypoxiaischemia is apoptosis. J Neurosci 21: 1931-1938, 2001.
5. Stumm RK, Rummel J, Junker V, Culmsee C, Pfeiffer M, Krieglstein J, Höllt V and Schulz S: A dual role for the SDF-1/CXCR4 chemokine receptor system in adult brain: Isoform-selective regulation of SDF-1 expression modulates CXCR4-dependent neuronal plasticity and cerebral leukocyte recruitment after focal ischemia. J Neurosci 22: 5865-5878, 2002.

6. Shyu WC, Lin SZ, Yen PS, Su CY, Chen DC, Wang HJ and Li H: Stromal cell-derived factor-1 alpha promotes neuroprotection, angiogenesis, and mobilization/homing of bone marrow-derived cells in stroke rats. J Pharmacol Exp Ther 324: 834-849, 2008.

7. Andiné P, Thordstein M, Kjellmer I, Nordborg C, Thiringer K, Wennberg $\mathrm{E}$ and Hagberg $\mathrm{H}$ : Evaluation of brain damage in a rat model of neonatal hypoxic-ischemia. J Neurosci Methods 35: 253-260, 1990.

8. Johnston MV: Neurotransmitter alterations in a model of perinatal hypoxic-ischemic brain injury. Ann Neurol 13: 511-518, 1983.

9. Ren C, Du A, Li D, Sui J, Mayhan WG and Zhao H: Dynamic change of hydrogen sulfide during global cerebral ischemiareperfusion and its effect in rats. Brain Res 1345: 197-205, 2010

10. Kilicdag H, Daglioglu YK, Erdogan S and Zorludemir S: Effects of caffeine on neuronal apoptosis in neonatal hypoxic-ischemic brain injury. J Matern Fetal Neonatal Med 27: 1470-1475, 2014.

11. Zhu M, Lu M, Li QJ, Zhang Z, Wu ZZ, Li J, Qian L, Xu Y and Wang ZY: Hyperbaric oxygen suppresses hypoxic-ischemic brain damage in newborn rats. J Child Neurol 30: 75-82, 2015.

12. Rice JE III, Vannucci RC and Brierley JB: The influence of immaturity on hypoxic-ischemic brain damage in the rat. Ann Neurol 9: 131-141, 1981

13. Levine S: Anoxic-ischemic encephalopathy in rats. Am J Pathol 36: 1-17, 1960.

14. Cowan CM, Thai J, Krajewski S, Reed JC, Nicholson DW, Kaufmann SH and Roskams AJ: Caspases 3 and 9 send a proapoptotic signal from synapse to cell body in olfactory receptor neurons. J Neurosci 21: 7099-7109, 2001.

15. Berger R and Garnier Y: Perinatal brain injury. J Perinat Med 28: 261-285, 2000.

16. Cheng Y, Deshmukh M, D'Costa A, Demaro JA, Gidday JM, Shah A, Sun Y, Jacquin MF, Johnson EM and Holtzman DM: Caspase inhibitor affords neuroprotection with delayed administration in a rat model of neonatal hypoxic-ischemic brain injury. J Clin Invest 101: 1992-1999, 1998.

17. Wang J, Van De Water TR, Bonny C, de Ribaupierre F, Puel JL and Zine A: A peptide inhibitor of c-Jun N-terminal kinase protects against both aminoglycoside and acoustic traumainduced auditory hair cell death and hearing loss. J Neurosci 23: 8596-8607, 2003.

18. Han W, Sun Y, Wang X, Zhu C and Blomgren K: Delayed, long-term administration of the caspase inhibitor Q-VD-OPh reduced brain injury induced by neonatal hypoxia-ischemia. Dev Neurosci 36: 64-72, 2014.

19. Wang X, Guo S, Lu S, Zhou J,Li J and Xia S: Ultrasound-induced release of GDNF from lipid coated microbubbles injected into striatum reduces hypoxic-ischemic injury in neonatal rats. Brain Res Bull 88: 495-500, 2012.

20. Xiao A, Kang M, Zhang $\mathrm{H}$ and Gong L: Effects of moxibustion pretreatment on caspase-3 expression in cortex of rats with cerebral ischemia-reperfusion injury. Tianjin Zhong Yi Yao 42: 550-552, 2014 (In Chinese).

This work is licensed under a Creative Commons Attribution-NonCommercial-NoDerivatives 4.0 International (CC BY-NC-ND 4.0) License. 